\title{
Tumor type-specific and skin region-selective metastasis of human cancers: another example of the "seed and soil" hypothesis
}

\author{
K. A. Kovács • B. Hegedus • I. Kenessey • J. Tímár \\ Published online: 1 May 2013 \\ (C) Springer Science+Business Media New York 2013
}

\begin{abstract}
Metastasis of human cancer is an organ-selective process that is determined by anatomical and biological factors as well as by specific microenvironmental properties. Dissemination of visceral malignancies to the skin is rather rare and usually occurs in a later stage of the disease. Using statistical approaches, both positive (renal and lung cancers) and negative (pancreatic and liver cancers) organ preferences can be identified in a variety of cancers. While certain cancer types are characterized by random distribution for skin metastasis (liver cancer), a number of cancers demonstrate a colonization preference to the region of origin: lung cancer to the supradiaphragmatic (mostly chest) and colorectal cancers to the infradiaphragmatic (abdominal) skin regions. In certain cases, however, skin metastasis develops more frequently at specific distant locations, as evidenced by the dissemination of
\end{abstract}

K.A. Kovács and B. Hegedus contributed equally to this work.

\section{Homage a Paget}

Electronic supplementary material The online version of this article (doi:10.1007/s10555-013-9418-8) contains supplementary material, which is available to authorized users.

K. A. Kovács • I. Kenessey • J. Tímár $(\bowtie)$

2nd Department of Pathology, Semmelweis University, Üllői u. 93, Budapest 1091, Hungary

e-mail: jtimar@gmail.com

K. A. Kovács

e-mail: attila.drkovacs@gmail.com

I. Kenessey

e-mail: steveken12@yahoo.com

B. Hegedus · J. Tímár

HAS-SU Tumor Progression Research Group,

Hungarian Academy of Sciences, Budapest, Hungary

B. Hegedus

e-mail: hegbal@yahoo.com

B. Hegedus

Department of Thoracic Surgery, Medical University of Vienna,

Vienna, Austria renal cancer at the head and neck region. These findings are clinically relevant and useful especially in patients where skin metastasis is the first indication of a malignancy. Nevertheless, it is a strong argument for the predominant role of microenvironmental factors in cancer dissemination. On the other hand, skin metastases of visceral cancers provide a unique model to analyze the pathomechanisms determining organ selectivity, including the organ-specific vascularization, the dermatome-specific innervation, or immunological and developmental factors.

Keywords Skin metastasis · Visceral organs $\cdot$ Regional distribution $\cdot$ Pathomechanisms

\section{Introduction}

Malignant progression of tumors is characterized by local invasion, lymphatic spreading to regional lymph nodes, and/or systemic hematogenous dissemination to distant tissues and organs described as the metastatic cascade [1]. In most cases, the survival of patients after successful eradication of the primary cancer depends on the development of distant metastasis through hematogenous dissemination. While local invasion and locoregional lymphatic spreading are biologically and pathologically predictable, the hematogenous dissemination is a rather stochastic process. There are two major pathomechanisms hypothesized that determine the target organ of hematogenous metastasis: Paget's "seed and soil hypothesis" [2] and Ewing's mechanical/anatomical theory [3]. Tumor cells can leave the primary tumor site through the venous drainage of the organ and this would predict that the first metastatic foci would develop in the filtering organ. This anatomical constrain could explain the frequent liver metastasis of the colorectal cancer and the lung metastasis of anal and lower rectal/anal cancers. Both preclinical and clinical studies demonstrated that circulating cancer cells can be detected at high frequency in the draining veins of a given primary cancer $[4,5]$. Nevertheless, distant metastasis is a highly inefficient 
(estimated at a rate of $0.01 \%$ ) and-importantly-highly selective process [6, 7]. Typical examples of high selectivity are bone and brain metastases of breast cancer, visceral metastases of malignant melanoma, or suprarenal gland metastasis of lung cancer. The metastatic colonization potential of a cancer depends on the genotype and phenotype of the cancer cells that determines its capacity to adapt to and/or manipulate the host organ microenvironment to support its survival, proliferation, vascularization, and ultimately metastasis formation [8].

\section{Skin as a rare metastatic site of cancers}

Skin is a relatively rare distant metastatic site of malignant tumors. Studies performed during the past 50 years found that about 1 to $5 \%$ of all visceral malignancies form skin metastasis [9]. Nevertheless, the prevalence may increase due to the longer survival of cancer patients [10-12]. Although skin is usually involved in a later stage of progression, it is the first sign of dissemination in a small fraction of cases [13-15]. However, there are two key factors that warrant special attention in skin metastasis statistics. First, a significant proportion of skin metastases are derived from cancer types that are closely connected to the skin such as melanoma, breast, or head and neck cancers [13-15]. Second, skin metastasis statistics describe natural frequencies and do not normalize the incidence with respect to the significant differences in the prevalence of the various cancer types. In order to establish the real organ preference of various cancers, skin metastasis numbers need to be adjusted with the relative prevalence of the specific tumor type. Of note, certain locations are subject to iatrogenic cell spreading such as the surgical scars in gastrointestinal cancer that can also influence the actual incidence of skin metastasis [16].

In this review, we intend to revisit the skin selectivity of metastasis of visceral cancer types and analyze their anatomical distribution based on literature data.

\section{Relative prevalence of skin metastasis of internal cancers}

The rate of skin metastases of various visceral cancers reported in previous studies is summarized in Table 1. There are three studies where the rate of skin metastasis for the majority of visceral cancers can be extracted [11, 17, 18]. We excluded breast and head and neck region (oral cavity, nasal sinuses, and larynx) cancers since these tumors have direct anatomical connection to the skin and previous studies have shown their high preference to skin metastasis. In order to establish the relative preference of the visceral cancer types to form skin metastasis, in each series, it is possible to calculate the primary tumor-specific rate of cutaneous metastasis in relation to the overall rate of skin metastasis in a given cohort. Another possibility for normalization is to use the specific incidence or mortality data for the given country and time period [19-21]. Due to the composition of the individual patient population in a given institution, this approach provides less consistent findings (Supplemental Table 1). Therefore, we averaged these relative rates over the three different cohorts. Using this approach, we identified two cancer types, namely, renal and lung cancers, with a high positive preference for the skin, while we found pancreatic and liver cancers with a high negative preference (Fig. 1). These data also indicate that colorectal, gastric, and genitourinary cancers are characterized by average skin metastatic preferences.

\section{Anatomical distribution of skin metastases of visceral cancers}

The role of local anatomical factors in the formation of skin metastasis of primary tumors that develop in the vicinity of the skin or within the skin is plausible. In contrast, in case of skin metastases of visceral organs, the role of anatomical factors is more complex. In Table 2, we summarized the various skin regions as metastatic locations of visceral cancers from previous studies [13, 18, 22, 23]. Based on these reports, the most common anatomical locations of skin metastases are the head and neck, chest, and abdominal regions. There is a rather large variation in case of the extremities due to the rather varying ratio of the different primary tumor types.

We performed a meta-analysis on 174 skin metastatic cases reported in five different studies [15, 18, 22-24] to identify location preferences of the primary tumors from different organs (Table 3). Importantly, lung, colorectal, and urogenital cancers displayed a preference to metastasize to their

Table 1 Rate of cutaneous metastasis of visceral cancers

\begin{tabular}{|c|c|c|c|c|c|c|c|c|}
\hline $\begin{array}{l}\text { Overall rate, } \\
\% \text { (no. of cases) }\end{array}$ & $\begin{array}{l}\text { Colorectal } \\
(\%)\end{array}$ & $\begin{array}{l}\text { Gastric } \\
(\%)\end{array}$ & $\begin{array}{l}\text { Lung } \\
(\%)\end{array}$ & $\begin{array}{l}\text { Renal } \\
(\%)\end{array}$ & $\begin{array}{l}\text { Pancreas } \\
(\%)\end{array}$ & $\begin{array}{l}\text { Liver } \\
(\%)\end{array}$ & $\begin{array}{l}\text { Genitourinary } \\
(\%)\end{array}$ & Reference \\
\hline $3.0(2,247)$ & 4.4 & 2.0 & 2.6 & 4.6 & 1.9 & - & 2.6 & [11] \\
\hline $0.8(6,477)$ & 0.8 & 0.8 & 1.8 & - & - & 0.3 & 0.3 & [17] \\
\hline $5.4(1,449)$ & 7.0 & 4.9 & 7.6 & 8.0 & 1.1 & 3.8 & 5.5 & [18] \\
\hline
\end{tabular}

Data are expressed in percent of the number of respective cancer cases 


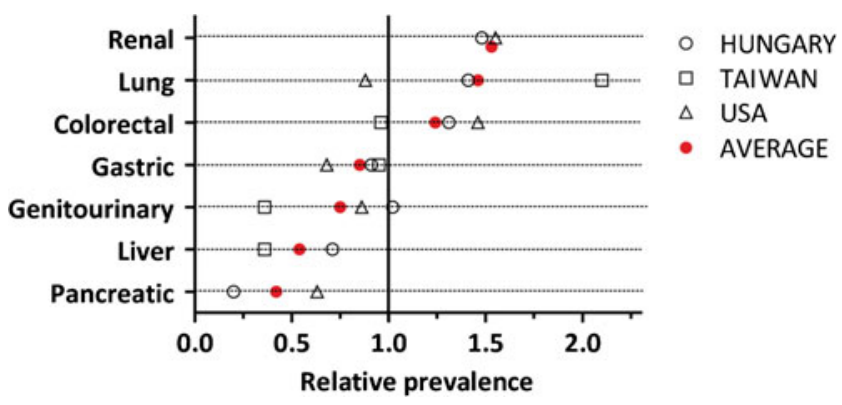

Fig. 1 Relative preference for skin metastases in various visceral cancers from three studies $[11,17,18]$. Primary tumor-specific relative rate was calculated according to the corresponding average rate from the study. If the number is $>1$, it describes a positive preference for skin metastasis formation

corresponding supradiaphragmatic and infradiaphragmatic skin regions. In contrast, skin metastasis of upper gastrointestinal tumors did not retain the original infradiaphragmatic position of the primary cancer, while skin metastases of renal cancer switched to the supradiaphragmatic region (Table 3).

To further support the observations above, we compiled 444 skin metastasis cases from 7 previous reports with different primary tumors and corresponding regional distribution data $[10,11,15,18,22-24]$ and from 2 lung cancer-specific studies $[25,26]$. These data strongly support the notion that lung cancer preferentially produces chest (and less frequently head and neck) skin metastases, while colorectal cancer prefers abdominal skin locations in more than two thirds of the cases (Fig. 2). Although the cohort is more limited for kidney cancer, a preference to head and neck region skin metastases was evident. Interestingly, liver cancer showed a random distribution pattern of skin metastasis (Fig. 2).

\section{Pathomechanisms underlying skin metastasis location preference}

\subsection{Vascular anatomy and lymphovascular dissemination}

There are three major types of skin metastases, namely, locoregional, in-transit, and distant ones. Melanoma and primary tumors in proximity to the skin (e.g., breast or head and

Table 2 Distribution of cutaneous metastasis in skin regions

\begin{tabular}{lllll}
\hline Region & $\begin{array}{l}n=80 \\
{[18](\%)}\end{array}$ & $\begin{array}{l}n=71 \\
{[13](\%)}\end{array}$ & $\begin{array}{l}n=57 \\
{[23](\%)}\end{array}$ & $\begin{array}{l}n=78 \\
{[22](\%)}\end{array}$ \\
\hline Head and neck & 21 & 42 & 37 & 25 \\
Chest & 25 & 21 & 14 & 8 \\
Abdomen & 44 & 30 & 25 & 26 \\
Extremities & 5 & 6 & 25 & 31 \\
\hline
\end{tabular}

The total number of cutaneous metastasis and the reference to the study are given in the column head. Data are expressed as percent of total cutaneous metastases in the given study neck cancer) preferentially produce skin metastases by locoregional spreading using mostly the lymphatic dissemination route [10, 11, 13-15]. In contrast, the mechanism for skin metastasis tumors residing in the body cavities is far less understood. With regards to the region-specific blood supply of the skin, head and neck skin regions are supplied by branches of external carotid and subclavian arteries, respectively, the skin of the trunk is supplied by spinal arteries, while the skin of the extremities are supplied from branches of their arteries derived from the lower aorta. However, this arterial blood supply heterogeneity does not explain regional variations in skin metastases either. There is no data indicating a direct connection of visceral organs and skin through lymphatic or blood vasculature. The one exception is the periumbilical skin region which is directly connected to abdominal organs through the umbilical artery. Accordingly, abdominal cancers at the stage of peritoneal dissemination may metastasize into the umbilicus also called Sister Mary Joseph's nodule [27]. The primary tumor most frequently is ovarian carcinoma in women and gastric cancer in men [28]. Despite the direct blood vessel connection, such type of metastasis is rare among skin metastases [27, 28]. Furthermore, in our recent report on abdominal skin metastases of gastrointestinal cancers, we found no association with peritoneal involvement [18]. Similarly, skin metastases of lung cancers are not associated with pleural involvement either suggesting that peritoneal or pleural spreading does not facilitate visceral cancer metastasis to the skin [18].

There are only two studies analyzing the lymphovascular invasion of skin metastases that could provide insight into the pathomechanism. A previous report found a $60-75 \%$ lymphovascular invasion rate in skin metastases [22]. However, the cohort contained predominantly melanoma, breast, and hematopoietic cancer cases that are known to prefer lymphatic dissemination. In a study containing exclusively visceral cancers, the lymphovascular invasion rate was much lower $(28.6 \%)$ and the majority of tumors contained lymphatic invasions [18]. Accordingly, locoregional spreading through lymphatics and the involvement of the corresponding peritoneal or pleural cavities do not represent the major pathomechanism of skin metastasis of visceral cancers.

\subsection{Blood perfusion rate}

The skin is one of the largest organs of the body, but its share from the cardiac output of blood is rather low: around $5 \%$ under resting conditions [29]. Preclinical studies indicated that intracardiac injection of melanoma cells into mice followed the distribution of blood in tissues and the rate of skin metastasis was $5 \%$ [30]. In sharp contrast, in humans, the incidence of skin metastasis is rather low $(<5 \%$ in our series) and lower than expected from blood flow share, indicating a general negative preference for this organ by various cancers 
Table 3 Distribution of skin metastases in relation to the diaphragm (meta-analysis)

\begin{tabular}{lllll}
\hline Skin metastasis $(n=174)$ & Diaphragm & Number & Percent & $p\left(\chi^{2}\right)$ \\
\hline Colorectal cancer $(n=48)$ & Supra & 16 & 33.3 & $<0.001$ \\
& Infra & 32 & 66.6 & \\
Upper gastrointestinal cancers $(n=25)$ & Supra & 12 & 48.0 & 0.365 \\
& Infra & 13 & 52.0 & \\
Lung cancer $(n=45)$ & Supra & 39 & 86.7 & $<0.0002$ \\
Renal cancer $(n=31)$ & Infra & 6 & 13.3 & \\
Other urogenital cancers $(n=25)$ & Supra & 26 & 83.9 & $<0.0002$ \\
& Infra & 5 & 16.1 & \\
& Supra & 5 & 20.0 & $<0.0001$ \\
\hline
\end{tabular}

[18]. There are three major factors that need to be considered to evaluate the skin as a host during metastasis formation. The skin, and the dermis in particular, is a very active part of both the antigen-specific and innate immune system that can interfere with the metastatic process. On the other hand, the skin is one of the most capillarized tissues in humans with a density of 70-90 capillaries per $\mathrm{mm}^{2}[31,32]$. Considering the spatial limits of oxygen and nutrient diffusion (around $100 \mu \mathrm{m}$ ), disseminated cancer cells in the skin do not depend on their own angiogenic potential. In conclusion, the relatively low perfusion rate and the active immunity seem to be obstacles for metastatic dissemination despite the abundance of nutrition and oxygen in the skin.

\subsection{Circulating cancer cells}

Circulating tumor cells (CTC) can readily be detected in almost all human malignant diseases and carry the potential

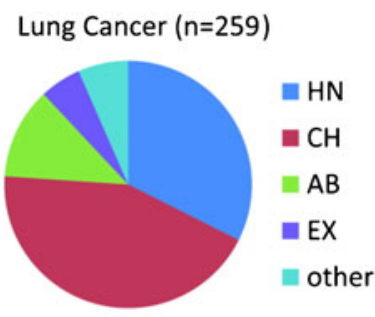

$P<0.0001 ;[10,15,18,22,24,26,27]$

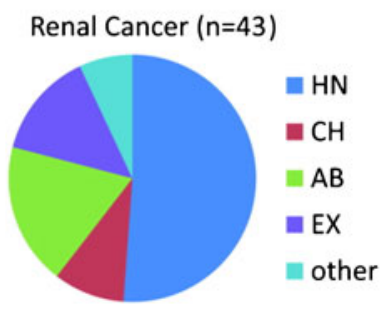

$P=0.026 ;[10,15,18,25]$
Colorectal Cancer $(n=132)$

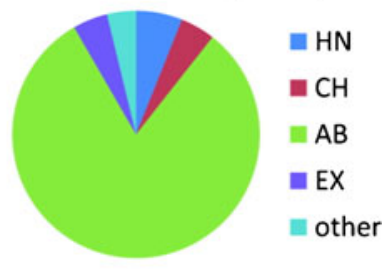

$\mathrm{P}<0.0001 ; \quad[10,15,18,22,24]$

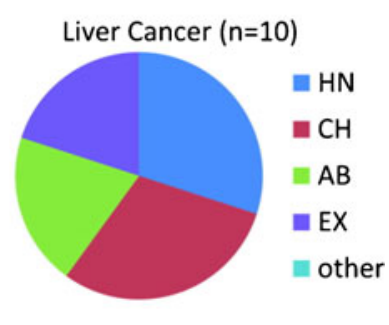

$P=0.66 ;[15,18]$
Fig. 2 Regional distribution of skin metastases of visceral cancers $(n=444)$. The meta-analysis is based on reports as indicated. The statistical significance of difference from a random distribution was determined by $\chi^{2}$ test. $H N$ head and neck, $C H$ chest, $A B$ abdominal, $E X$ extremity to become metastatic cells [33-35]. Importantly, they can be detected with a relatively high incidence even in early stage disease [34]. Reports on the comparison of early and late stage disease CTC numbers found limited correlation [36, 37]. With advances of cell isolation technologies, the detection threshold has become lower, reaching 1-5 CTC/10 ml blood [32]. It is a well-established fact that the metastatic efficiency of hematogenous dissemination is $0.01 \%$; in other words, 1 cell out of 10,000 tumor cells in the circulation $[6,7]$, indicating that the overwhelming majority of CTC is incapable of colonizing the distant organs. Upon further consideration of this fact in the era of CTC isolation technologies, one can state that CTC positivity $(5 / 10 \mathrm{ml})$ means $2,500 \mathrm{CTC}$ in the whole blood volume in a given moment in a cancer patient. Regarding the $1 / 10^{4}$ efficacy rate of cancer cells to metastasize, this low number of CTC is not necessarily enough for producing a single metastatic colony. However, this calculation must be corrected with the share of blood flow of the individual metastatic organ. In case of the skin, the $5 \%$ means 1/20 of the entire blood volume, meaning that 20 times more CTC is necessary to produce 1 single skin colony (equivalent of 250 $500 \mathrm{CTC} / 10 \mathrm{ml}$ ), a very high number that is rarely detected in humans [33-35]. Due to the aforementioned obstacles in the skin microenvironment and the low perfusion rate, the colonization of the skin requires a rather high CTC number that occurs only in a limited number of cancer cases and in general at late stages.

\subsection{Mechanisms of organ selectivity}

Statistical analysis of our and two other datasets indicated that pancreatic and liver cancers show the highest negative preference and that renal and lung cancers show the strongest positive preference to skin metastasis (Fig. 1). In case of the abdominal skin metastasis of colorectal cancer, the direct umbilical vascular connection may contribute to the preference; however, this type of metastasis is rather infrequent.

Endothelial cells in capillary beds express unique organspecific markers that have previously been identified by 
antibody-based [38], lectin-binding [39], phage display [40], or proteomic technologies [41]. Furthermore, a skinspecific marker with a defined peptide sequence (CVALCREACGEGC) has been identified that functions as a selective adhesion partner for circulating cancer cells for extravasation [42]. On the other hand, skin-homing cancer cells must recognize dermal tissues in a highly specific way. Studies on human melanomas revealed that these epidermotropic cancer cells express CCR10 chemokine receptor which is used for skin homing [43]. The ligand for CCR10 is a skin-specific keratinocyte chemokine, CCL27 [44]. In the dermis, fibroblasts and endothelial cells secrete SDF1/CXCL12 and its receptor, CXCR4, is present on skinhoming Sezary lymphoma cells [45]. There has been an exploratory analysis on three breast and three lung cancer cases: CCR10 and CXCR4 expressions were determined in primary tumors with and without skin metastases [17]. CXCR4 was readily expressed in both primary and skin metastatic tumors of both breast and lung cancer. CCR10 was variably present in both breast and lung primary tumors but was only present in skin metastases of breast cancers but not of lung cancer [17]. Since only the minority of skin metastases of visceral organ cancers is epidermotropic [13], CCR10 expression might not be crucial for skin-homing for most cancer types unlike CXCR4. Furthermore, a recent study found that CXCR4/CXCL12 plays a significant role in pancreatic cancer organ-specific metastasis [46]. However, in renal cancer, the incidence of CXCR4 expression is lower $[47,48]$ than in colorectal $[49,50]$ or pancreatic cancers [51,52], suggesting that this chemokine system may not be the primary driver of the skin-selective homing mechanism.

\subsection{Mechanisms underlying regional distribution}

Blood circulation-dependent tumor spreading would indicate a randomly distributed skin metastasis pattern, but there is evidence that most visceral tumors have preferred skin regions for metastasis. Random skin metastasis pattern characterizes liver cancer dissemination, but this is an exception. Most skin metastases develop in a nonrandom manner. Several previous reports [10, 11, 13-15, 18] indicated that significantly more skin metastases occur in the ventral skin. Furthermore, we provided new statistical evidence that some visceral cancer types gave metastases to the skin regions of the "same level," such as lung cancer mainly to supradiaphragmatic (primarily chest), colorectal cancer mainly to infradiaphragmatic (primarily abdominal), and genitourinary cancers to lower abdominal sites, as suggested by earlier observations $[10,11,13-15]$. It is also of note that skin metastases do not respect the laterality of the primary tumor [18]. On the other hand, in case of certain visceral cancers, we have found a peculiar nonregional skin preference, e.g., the head and neck localization of kidney cancer metastases. These observations are strong arguments for the existence of certain "homing" mechanisms that determine the terminal point of CTC in the skin tissue.

Although being a unified organ, the skin of the body is characterized by regional anatomical/histological differences in respect to hair density, epidermal characteristics, thickness of the dermis, and amount of underlying adipose tissue. It is another heterogeneity factor that skin regions can be divided into 30 dermatomes based on the spinal nerve innervation [53]. While these dermatomes are highly uniform in the thoracic and abdominal regions, they are very complex on the extremities. It is intriguing that certain cancers (such as lung or colon cancer) follow their dermatomal segments in skin metastasis (T1-T7 and T8-L1, respectively), while others such as renal cancers prefer the cervical dermatomes. Perineural invasion of various cancer types (breast, colorectal, pancreatic, gastric, prostate, or head and neck cancers) is a common feature in the primary tumors with prognostic significance. Meanwhile in our cohort, we did not find perineural invasion in skin metastases [18] and earlier pathological analyses have not mentioned them either $[10,11,13-15]$, making a peripheral nerve association of skin metastasis development highly unlikely. Skin is also patterned by a developmental segmentation called Blaschko lines that do not follow nerves, blood vessels, or lymphatics, though they are similar to dermatomes [54]. Certain skin diseases clearly follow such embryonal patterns as lichen or nevoid disorders [54]. Since the molecular biological basis of these skin patterns is not known yet, it is impossible to speculate further concerning the "seed and soil" hypothesis of skin metastasis formation.

\section{Conclusion}

Skin metastasis of visceral cancers is another strong proof for the validity of the seed and soil hypothesis, i.e., the organ-selective nature of the metastasis formation process. The molecular basis of this selective process is largely unknown, although in case of certain organs such as the lung or the bone, basic mechanisms are defined. In general, it can be concluded that skin metastasis is a rare form of cancer dissemination due to the unique physiology of this organ. On the other hand, if it occurs, it is frequently a nonrandom process which can be used for the identification of the primary tumor in the event this is the first sign of the disease. Among the various cancer types, there are several which exhibit positive or negative preferences for skin colonization even in an advanced late stage. Identification of 
the underlying molecular mechanisms behind this process can also contribute to better understanding of the cancer dissemination process in general.

\section{References}

1. Liotta, L. A. (1986). Tumor invasion and metastases-Role of the extracellular matrix: Rhoads Memorial Award lecture. Cancer Research, 46(1), 1-7.

2. Paget, S. (1889). The distribution of secondary growth in cancer of the breast. Lancet, 133, 571-573.

3. Ewing, J. (1928). Neoplastic diseases: A treatise on tumors (3rd ed.). Philadelphia: W.B. Saunders (rev. and enl., with 546 illustrations).

4. Sienel, W., Seen-Hibler, R., Mutschler, W., Pantel, K., \& Passlick, B. (2003). Tumour cells in the tumour draining vein of patients with non-small cell lung cancer: Detection rate and clinical significance. European Journal of Cardio-Thoracic Surgery: Official Journal of the European Association for Cardio-Thoracic Surgery, 23(4), 451-456.

5. Rahbari, N. N., Bork, U., Kircher, A., Nimitz, T., Scholch, S., Kahlert, C., et al. (2012). Compartmental differences of circulating tumor cells in colorectal cancer. Annals of Surgical Oncology, 19(7), 2195-2202.

6. Langley, R. R., \& Fidler, I. J. (2011). The seed and soil hypothesis revisited-The role of tumor-stroma interactions in metastasis to different organs. International Journal of Cancer, 128(11), 25272535.

7. Talmadge, J. E., \& Fidler, I. J. (2010). AACR centennial series: The biology of cancer metastasis: Historical perspective. Cancer Research, 70(14), 5649-5669.

8. Hanahan, D., \& Weinberg, R. A. (2011). Hallmarks of cancer: The next generation. Cell, 144(5), 646-674.

9. Mueller, T. J., Wu, H., Greenberg, R. E., Hudes, G., Topham, N., Lessin, S. R., et al. (2004). Cutaneous metastases from genitourinary malignancies. Urology, 63(6), 1021-1026.

10. Brownstein, M. H., \& Helwig, E. B. (1972). Patterns of cutaneous metastasis. Archives of Dermatology, 105(6), 862-868.

11. Lookingbill, D. P., Spangler, N., \& Helm, K. F. (1993). Cutaneous metastases in patients with metastatic carcinoma: A retrospective study of 4020 patients. Journal of the American Academy of Dermatology, 29(2 Pt 1), 228-236.

12. Brownstein, M. H., \& Helwig, E. B. (1972). Metastatic tumors of the skin. Cancer, 29(5), 1298-1307.

13. Alcaraz, I., Cerroni, L., Rutten, A., Kutzner, H., \& Requena, L. (2012). Cutaneous metastases from internal malignancies: A clinicopathologic and immunohistochemical review. American Journal of Dermatopathology, 34(4), 347-393.

14. Hussein, M. R. (2010). Skin metastasis: A pathologist's perspective. Journal of Cutaneous Pathology, 37(9), e1-e20.

15. Nashan, D., Muller, M. L., Braun-Falco, M., Reichenberger, S., Szeimies, R. M., \& Bruckner-Tuderman, L. (2009). Cutaneous metastases of visceral tumours: A review. Journal of Cancer Research and Clinical Oncology, 135(1), 1-14.

16. Alexandrescu, D. T., Vaillant, J., Yahr, L. J., Kelemen, P., \& Wiernik, P. H. (2005). Unusually large colon cancer cutaneous and subcutaneous metastases occurring in resection scars. Dermatology Online Journal, 11(2), 22.

17. Hu, S. C., Chen, G. S., Wu, C. S., Chai, C. Y., Chen, W. T., \& Lan, C. C. (2009). Rates of cutaneous metastases from different internal malignancies: Experience from a Taiwanese medical center. Journal of the American Academy of Dermatology, 60(3), 379-387.

18. Kovacs, K. A., Kenessey, I., Timar, J. (2013). Skin metastasis of internal cancers: A single institution experience. Pathology and Oncology Research. doi:10.1007/s12253-013-9611-7.

19. American Cancer Society (2005). Cancer facts and figures 2005. Retrieved from http://www.cancer.org/research/cancerfactsfigures/ cancerfactsfigures/cancer-facts-figures-2005.

20. Chiang, C. J., Chen, Y. C., Chen, C. J., You, S. L., \& Lai, M. S. (2010). Cancer trends in Taiwan. Japanese Journal of Clinical Oncology, 40(10), 897-904.

21. Kasler, M., \& Otto, S. (2008). European and Hungarian national tasks in oncology. Magyar Onkologia, 52(1), 21-33.

22. Fernandez-Flores, A. (2010). Cutaneous metastases: A study of 78 biopsies from 69 patients. American Journal of Dermatopathology, $32(3), 222-239$.

23. Saeed, S., Keehn, C. A., \& Morgan, M. B. (2004). Cutaneous metastasis: A clinical, pathological, and immunohistochemical appraisal. Journal of Cutaneous Pathology, 31(6), 419-430.

24. Dorairajan, L. N., Hemal, A. K., Aron, M., Rajeev, T. P., Nair, M., Seth, A., et al. (1999). Cutaneous metastases in renal cell carcinoma. Urologia Internationalis, 63(3), 164-167.

25. Kamble, R., Kumar, L., Kochupillai, V., Sharma, A., Sandhoo, M. S., \& Mohanti, B. K. (1995). Cutaneous metastases of lung cancer. Postgraduate Medical Journal, 71(842), 741-743.

26. Song, Z. B., Lin, B. C., Shao, L., \& Zhang, Y. P. (2012). Cutaneous metastasis as a initial presentation in advanced non-small cell lung cancer and its poor survival prognosis. Journal of Cancer Research and Clinical Oncology, 138(10), 1613-1617.

27. Pereira, W. A., Humaire, C. R., Silva, C. S., \& Fernandes, L. H. G. (2011). Sister Mary Joseph's nodule: A sign of internal malignancy. Anais Brasileiros De Dermatologia, 86(4), 118-120.

28. Dubreuil, A., Dompmartin, A., Barjot, P., Louvet, S., \& Leroy, D. (1998). Umbilical metastasis or Sister Mary Joseph's nodule. International Journal of Dermatology, 37(1), 7-13.

29. Calzia, E., Iványi, Z., Radermacher, P. (2005). Determinants of blood flow and organ perfusion. In: Pinsky, M., Payen, D. (eds.) Functional hemodynamic monitoring, vol. 42. Update in intensive care and emergency medicine (pp. 19-32). Berlin: Springer.

30. Weiss, L., Ward, P. M., Harlos, J. P., \& Holmes, J. C. (1984). Target organ patterns of tumors in mice following the arterial dissemination of B16 melanoma cells. International Journal of Cancer, 33(6), 825-830.

31. Dome, B., Paku, S., Somlai, B., \& Timar, J. (2002). Vascularization of cutaneous melanoma involves vessel co-option and has clinical significance. The Journal of Pathology, 197(3), 355-362.

32. Mork, C., Kvernebo, K., Asker, C. L., \& Salerud, E. G. (2002). Reduced skin capillary density during attacks of erythromelalgia implies arteriovenous shunting as pathogenetic mechanism. Journal of Investigative Dermatology, 119(4), 949-953.

33. Marrinucci, D., Bethel, K., Kolatkar, A., Luttgen, M. S., Malchiodi, M., Baehring, F., et al. (2012). Fluid biopsy in patients with metastatic prostate, pancreatic and breast cancers. Physical Biology, 9(1), 016003.

34. Sun, Y. F., Yang, X. R., Zhou, J., Qiu, S. J., Fan, J., \& Xu, Y. (2011). Circulating tumor cells: Advances in detection methods, biological issues, and clinical relevance. Journal of Cancer Research and Clinical Oncology, 137(8), 1151-1173.

35. Yu, M., Stott, S., Toner, M., Maheswaran, S., \& Haber, D. A. (2011). Circulating tumor cells: Approaches to isolation and characterization. The Journal of Cell Biology, 192(3), 373-382.

36. Wendel, M., Bazhenova, L., Boshuizen, R., Kolatkar, A., Honnatti, M., Cho, E. H., et al. (2012). Fluid biopsy for circulating tumor cell identification in patients with early- and late-stage non-small cell lung cancer: A glimpse into lung cancer biology. Physical Biology, 9(1), 016005. 
37. Nieva, J., Wendel, M., Luttgen, M. S., Marrinucci, D., Bazhenova, L., Kolatkar, A., et al. (2012). High-definition imaging of circulating tumor cells and associated cellular events in non-small cell lung cancer patients: A longitudinal analysis. Physical Biology, 9(1), 016004.

38. Ghitescu, L. D., Crine, P., \& Jacobson, B. S. (1997). Antibodies specific to the plasma membrane of rat lung microvascular endothelium. Experimental Cell Research, 232(1), 47-55.

39. Belloni, P. N., \& Nicolson, G. L. (1988). Differential expression of cell surface glycoproteins on various organ-derived microvascular endothelia and endothelial cell cultures. Journal of Cellular Physiology, 136(3), 398-410.

40. Pasqualini, R., \& Ruoslahti, E. (1996). Organ targeting in vivo using phage display peptide libraries. Nature, 380(6572), 364-366.

41. Simonson, A. B., \& Schnitzer, J. E. (2007). Vascular proteomic mapping in vivo. Journal of Thrombosis and Haemostasis, 5(Suppl 1), 183-187.

42. Rajotte, D., Arap, W., Hagedorn, M., Koivunen, E., Pasqualini, R., \& Ruoslahti, E. (1998). Molecular heterogeneity of the vascular endothelium revealed by in vivo phage display. The Journal of Clinical Investigation, 102(2), 430-437.

43. Murakami, T., Cardones, A. R., \& Hwang, S. T. (2004). Chemokine receptors and melanoma metastasis. Journal of Dermatological Science, 36(2), 71-78.

44. Xiong, N., Fu, Y., Hu, S., Xia, M., \& Yang, J. (2012). CCR10 and its ligands in regulation of epithelial immunity and diseases. Protein \& Cell, 3(8), 571-580.

45. Narducci, M. G., Scala, E., Bresin, A., Caprini, E., Picchio, M. C., Remotti, D., et al. (2006). Skin homing of Sezary cells involves SDF-1CXCR4 signaling and down-regulation of CD26/dipeptidylpeptidase IV. Blood, 107(3), 1108-1115.

46. Zhong, W., Chen, W., Zhang, D., Sun, J., Li, Y., Zhang, J., et al. (2012). CXCL12/CXCR4 axis plays pivotal roles in the organ- specific metastasis of pancreatic adenocarcinoma: A clinical study. Experimental and Therapeutic Medicine, 4(3), 363-369.

47. D'Alterio, C., Portella, L., Ottaiano, A., Rizzo, M., Carteni, G., Pignata, S., et al. (2012). High CXCR4 expression correlates with sunitinib poor response in metastatic renal cancer. Current Cancer Drug Targets, 12(6), 693-702.

48. Zhao, F. L., \& Guo, W. (2011). Expression of stromal derived factor-1 (SDF-1) and chemokine receptor (CXCR4) in bone metastasis of renal carcinoma. Molecular Biology Reports, 38(2), 1039-1045.

49. Sakai, N., Yoshidome, H., Shida, T., Kimura, F., Shimizu, H., Ohtsuka, M., et al. (2012). CXCR4/CXCL12 expression profile is associated with tumor microenvironment and clinical outcome of liver metastases of colorectal cancer. Clinical \& Experimental Metastasis, 29(2), 101-110.

50. Yopp, A. C., Shia, J., Butte, J. M., Allen, P. J., Fong, Y., Jarnagin, W. R., et al. (2012). CXCR4 expression predicts patient outcome and recurrence patterns after hepatic resection for colorectal liver metastases. Annals of Surgical Oncology, 19(Suppl 3), S339S346.

51. Gebauer, F., Tachezy, M., Effenberger, K., von Loga, K., Zander, H., Marx, A., et al. (2011). Prognostic impact of CXCR4 and CXCR7 expression in pancreatic adenocarcinoma. Journal of Surgical Oncology, 104(2), 140-145.

52. Marechal, R., Demetter, P., Nagy, N., Berton, A., Decaestecker, C., Polus, M., et al. (2009). High expression of CXCR4 may predict poor survival in resected pancreatic adenocarcinoma. British Journal of Cancer, 100(9), 1444-1451.

53. Lee, M. W. L., McPhee, R. W., \& Stringer, M. D. (2008). An evidence-based approach to human dermatomes. Clinical Anato$m y, 21(5), 363-373$.

54. Happle, R. (2002). Transposable elements and the lines of Blaschko: A new perspective. Dermatology, 204(1), 4-7. 